\title{
A Formula for the Maximum Vertical Velocity in Supercell Updrafts
}

\author{
John M. Peters,${ }^{\mathrm{a}}$ Hugh Morrison, ${ }^{\mathrm{b}, \mathrm{c}, \mathrm{d}}$ CHRISTOPHER J. NOWOTARSKI,${ }^{\mathrm{e}}$ \\ JAKE P. MULhOLLAND, ${ }^{\mathrm{a}}$ AND RiCHARD L. THOMPSON ${ }^{\mathrm{f}}$ \\ ${ }^{a}$ Department of Meteorology, Naval Postgraduate School, Monterey, California; ${ }^{\mathrm{b}}$ National Center for Atmospheric Research, \\ Boulder, Colorado; ${ }^{\mathrm{c}}$ Climate Change Research Centre, University of New South Wales, Sydney, New South Wales, Australia, \\ ${ }^{\mathrm{d}}$ Australian Research Council Centre for Excellence in Climate System Science, University of New South Wales, Sydney,

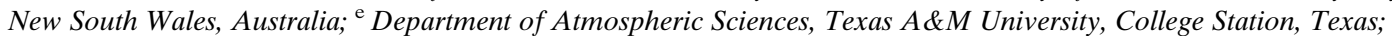 \\ ${ }^{\mathrm{f}} \mathrm{NOAA} / \mathrm{NWS} / \mathrm{NCEP} /$ Storm Prediction Center, Norman, Oklahoma
}

(Manuscript received 8 April 2020, in final form 18 July 2020)

\begin{abstract}
In supercell environments, previous authors have shown strong connections between the vertical wind shear magnitude, updraft width, and entrainment. Based on these results, it is hypothesized that the influences of entrainmentdriven dilution on buoyancy and maximum updraft vertical velocity $w$ in supercell environments are a predictable function of the vertical wind shear profile. It is also hypothesized that the influences of pressure perturbation forces on maximum updraft $w$ are small because of a nearly complete offset between upward dynamic pressure forces and downward buoyant pressure forces. To address these hypotheses, we derive a formula for the maximum updraft $w$ that incorporates the effects of entrainment-driven dilution on buoyancy but neglects pressure gradient forces. Solutions to this formula are compared with output from previous numerical simulations. This formula substantially improves predictions of maximum updraft $w$ over past CAPE-derived formulas for maximum updraft $w$, which supports the first hypothesis. Furthermore, integrated vertical accelerations along trajectories show substantial offsets between dynamic and buoyant pressure forces, supporting the second hypothesis. It is argued that the new formula should be used in addition to CAPE-derived measures for $w$ in forecast and research applications when accurate diagnosis of updraft speed is required.
\end{abstract}

KEYWORDS: Buoyancy; Deep convection; Entrainment; Severe storms; Supercells

\section{Introduction}

Supercell thunderstorms are responsible for a large percentage of damages related to large hail, excessive rainfall, damaging straight-line winds, and tornadoes in the central United States (e.g., Gallus et al. 2008). Many of these severe weather hazards are influenced by the strength of vertical velocities $w$ in updrafts. For instance, stronger updrafts produce larger hailstones than weaker updrafts (e.g., Browning 1963), downdrafts and the associated damaging wind potential are dynamically coupled with updrafts (e.g., Marion and Trapp 2019), precipitation production relates to vertical mass flux and consequently $w$ (Doswell et al. 1996), and vertical accelerations in the lower part of updrafts play a critical role in tornadogenesis (e.g., Coffer and Parker 2015).

Convective available potential energy (CAPE; e.g., Moncrieff and Miller 1976) has long been used as a general guide for predicting $w$ in supercell updrafts (e.g., Thompson et al. 2003, 2007; Smith et al. 2012; Thompson et al. 2012). This quantity assumes that the only force acting upon an air parcel is buoyancy and uses a vertical profile of atmospheric temperature and moisture to estimate the maximum vertical kinetic energy and $w$ for an air parcel ascending through a convective updraft. Calculations for CAPE are advantageous in that they only require a single atmospheric sounding, and consequently CAPE measures are readily used in forecasting and in cumulus parameterizations. However, the following well-known flaws limit the utility of CAPE as a forecast metric:

Corresponding author: J. Peters, jmpeters@nps.edu
1) Updraft properties are often substantially diluted by entrainment, which is neglected in CAPE calculations. Entrainment strongly modulates updraft buoyancy and consequently $w$ (e.g., Romps and Kuang 2010; Morrison 2017; Peters et al. 2019).

2) The formulation for CAPE neglects vertical perturbation pressure gradient forces, which are often substantial in deep convection, particularly supercells (e.g., Weisman and Klemp 1984; Davies-Jones 2003; Doswell and Markowski 2004; Morrison 2016a; Peters 2016; Tarshish et al. 2018).

Unfortunately, none of the aforementioned shortcomings are easily remedied given that clouds' entrainment and pressure perturbation properties are difficult to anticipate from a single sounding.

Many early conceptual studies of deep moist convection accounted for entrainment by invoking the canonical "entraining plume" model. This model originates from laboratory studies of dry convection and dimensional analysis (e.g., Morton et al. 1956; Morton 1957; Emanuel 1994). The model's estimate for $w$ improves upon parcel theory by accounting for the role of entrainment in diluting cloud properties. To simplify the treatment of entrainment in this model, traditional formulations assumed that fractional entrainment rates were constant with height and among cloud types (e.g., Arakawa and Schubert 1974; Zhang and McFarlane 1995). Given a simple assumed fractional entrainment rate profile, entraining plume models are used to produce modified entrainment CAPE (ECAPE) calculations that improve closure assumptions in cumulus parameterizations (e.g., Zhang and McFarlane 1991; Zhang 2009). However, it is well known that fractional entrainment 
rates exhibit complex vertical, spatial, and temporal variability (e.g., Romps 2010; Hannah 2017; Morrison et al. 2020; Peters et al. 2020a) suggesting that these models have oversimplified entrainment. Indeed, the so-called entrainment paradox plagued early versions of cumulus parameterizations, wherein no single fractional entrainment rate could optimally reproduce all aspects of cloud properties (e.g., Warner 1970; Cotton 1975). More recent versions of the entraining plume model have partially remedied the entrainment paradox by incorporating the buoyancy sorting concept (Zhao and Austin 2003), treating entrainment as a stochastic process (e.g., Romps 2016), and/or modifying entrainment rates based on heuristic (e.g., Mapes and Neale 2011) or empirical (e.g., Holloway and Neelin 2009; Ahmed and Neelin 2018) relationships between entrainment and environmental characteristics. However, despite these efforts our field has yet to converge on a unifying representation of entrainment that applies to deep moist convection among all environments, and for all applications.

Relative to the cumulus parameterization and climate dynamics communities, comparatively few attempts have been made to represent the influences of entrainment in forecasting diagnostics for severe convective weather. For instance, all of the currently available CAPE measures on the Storm Prediction Center mesoscale analysis website omit the influences of entrainment on air parcel buoyancy. These omissions are perhaps a result of the aforementioned difficulties in accurately representing entrainment rates over a wide range of convective environments, and/or because entrainment may be under appreciated relative to the often extreme CAPE and vertical wind shear that occur in severe storm environments. However, a recent study of tropical cyclone tornadoes in Tochimoto et al. (2019) showed that using ECAPE instead of standard CAPE calculations in forecasting allowed for better discrimination between tornadic and nontornadic tropical cyclone events. Additionally, our recent work has shown that entrainment substantially modifies the buoyancy of deep convective updrafts, leading to large differences in updraft buoyancy among environments that share identical CAPE (Peters et al. 2019). In fact, the results of that study suggest that entrainment rates are more predictable for a given atmospheric sounding in severe convective environments than their nonsevere counterparts. This is because supercell updraft width shows strong connections with deep-layer vertical wind shear and low-level storm-relative flow (e.g., Warren et al. 2017; Trapp et al. 2017; Peters et al. 2019; Marion and Trapp 2019). Furthermore, numerous studies have also shown that fractional entrainment is dependent on updraft width (e.g., Kyle et al. 1976; Morrison 2017; Lecoanet and Jeevanjee 2019; Hernandez-Deckers and Sherwood 2018; Peters et al. 2019, 2020b; Morrison et al. 2020; Peters et al. 2020a). Based on these connections, we hypothesize that predictions for supercell updraft buoyancy and vertical velocity may be improved by accounting for the aforementioned connections between environmental wind characteristics, updraft width, and entrainment (without the need for a prespecified entrainment rate). Addressing this hypothesis (hereinafter H1) is the first objective of this article.
Various methods have been used to represent pressure perturbation effects in entraining plume models in order to remedy the omission of these effects in parcel theory. Pressure perturbation effects were included in early plume models implicitly, by applying a constant fractional scaling parameter to the buoyancy term in the vertical momentum equation (Simpson and Wiggert 1969; Siebesma et al. 2003). On the other hand, several studies have shown that vertical pressure gradient forces substantially influence the shape of the vertical profile of $w$, suggesting that relegating these effects to a constant virtual mass coefficient was an oversimplification (e.g., De Roode et al. 2012; Sherwood et al. 2013; Romps and Charn 2015; Hernandez-Deckers and Sherwood 2016; Peters 2016; Morrison and Peters 2018; Tian et al. 2019). Our recent work has shown that for deep convection occurring in weak vertical wind shear, the updraft maximum $w$ is primarily determined by buoyancy and buoyancy pressure accelerations (e.g., Peters 2016; Morrison and Peters 2018). While dynamic pressure perturbations substantially influence the shape of the vertical profile of $w$, the overall contribution by dynamic pressure to the magnitude of maximum updraft $w$ was typically $10 \%$ or less. This result simplifies predictions for the maximum $w$ in updrafts in weakly sheared environments because such predictions do not require the determination of the dynamic pressure characteristics of updrafts because of the relatively small contributions from these pressure features.

In contrast with weakly sheared deep moist convection, vertical-wind shear induced pressure perturbations play a predominant role in supercell updraft dynamics (e.g., Weisman and Klemp 1984; Rotunno and Klemp 1982, 1985; McCaul and Weisman 1996; Weisman and Rotunno 2000; Davies-Jones 2002; Peters et al. 2019). Furthermore, numerous previous studies have shown substantial contributions to the maximum updraft $w$ from dynamic pressure accelerations in supercells (e.g., Weisman and Klemp 1982; McCaul and Weisman 1996; Weisman and Rotunno 2000; Peters et al. 2019), suggesting that the conclusions regarding the minimal role of dynamic pressure accelerations in weakly sheared deep moist convection do not apply to supercells. However, results from Peters et al. (2019) show that the magnitudes of both upward directed dynamic pressure accelerations and downward directed buoyant pressure accelerations substantially offset each other. It is therefore reasonable to hypothesize that we may simply neglect pressure perturbation effects altogether in predicting maximum updraft $w$ in supercells (hereinafter H2). Although pressure perturbations have substantial influences on thunderstorm evolution, their influence on the overall maximum $w$ is minimal because of the aforementioned offsetting effect. Such a result would certainly be convenient from a forecasting perspective, since it would greatly simplify the task of predicting maximum updraft $w$ in supercells.

The purpose of this article is to use a combination of theory and numerical simulations to address $\mathrm{H} 1$ and $\mathrm{H} 2$, in order to improve our ability to predict maximum updraft $w$ from an atmospheric sounding in forecasting and research applications that rely on this quantity. A theoretical expression for the maximum updraft $w$ in supercell environments as a function of environmental CAPE and vertical wind shear is derived in 
section 2. This expression is formulated from the equations of motion and assumptions based on known updraft behavior and is shown to improve skill in predicting maximum updraft $w$ over traditional and CAPE and ECAPE measures. The behavior of this expression in the parameter space of CAPE and vertical wind shear is explored in section 3 . The new expression is then compared with numerical simulations of deep moist convection to validate the assumptions used to derive the expression in section 4 , and a summary, conclusions, and discussion are provided in section 5 .

\section{Derivation of theoretical expression}

We begin our derivation with the Boussinesq ${ }^{1}$ vertical momentum equation, which is written as

$$
\frac{D w}{D t}=B-\frac{1}{\rho_{0}} \frac{\partial p_{B}}{\partial z}-\frac{1}{\rho_{0}} \frac{\partial p_{D}}{\partial z},
$$

where $B$ is buoyancy, BPA stands for buoyancy pressure acceleration, and DPA stands for dynamic pressure acceleration. The individual pressure terms are defined via the following equations: ${ }^{2}$

$$
\nabla^{2} p_{B}=\frac{\partial\left(\rho_{0} B\right)}{\partial z} \text { and } \nabla^{2} p_{D}=-\rho_{0} \nabla \cdot[(\mathbf{V} \cdot \nabla) \mathbf{V}]
$$

where $\mathbf{V}$ is the three-dimensional wind, $\rho_{0}$ is the invariant background density, $p_{B}$ is "buoyancy pressure" and $p_{D}$ is " dynamic pressure."

Assuming a steady state such that $D w / D t \approx(1 / 2) \partial w^{2} / \partial z$ and vertically integrating from the surface to the height $H$ of maximum $w$ gives

$$
w^{2}=w_{B}^{2}-2 \frac{\Delta p_{B}}{\rho_{0}}-2 \frac{\Delta p_{D}}{\rho_{0}},
$$

where $w_{B}^{2} \equiv 2 \int_{z=0}^{z=H} B d z$ and $\Delta$ denotes the vertical difference in a quantity from the surface to $H$. At this stage, there are four unknown parameters $\left(w, w_{B}, \Delta p_{B}\right.$, and $\left.\Delta p_{D}\right)$ that are not easily computed from a given atmospheric sounding.

To parameterize the influence of entrainment on $B$, Morrison (2017) used an eddy diffusivity approximation for the horizontal mixing of ambient environmental air with updraft core air. He assumed a constant saturation of $100 \%$ during moist ascent and neglected the latent heat of fusion

\footnotetext{
${ }^{1}$ This assumption leads to errors in the computation of the pressure field; however, such errors are minimized in the context of $w$ because pressure gradients are divided by density to calculate vertical pressure gradient forces (e.g., Morrison 2016a; Peters 2016; Peters et al. 2019).

${ }^{2}$ Numerical solutions for these terms were obtained from model data by solving for the right-hand-side terms, applying a horizontal 2D Fourier transform in the horizontal direction using the method of images to enforce zero-gradient lateral boundary conditions (e.g., Davies-Jones 2002), solving the resultant tridiagonal matrix equation, and then inverting the $2 \mathrm{D}$ horizontal Fourier transform.
}

to obtain the following expression for cloud core $B$ as a function of $z$ :

$$
B=B_{\mathrm{AD}}+\frac{L}{R^{2}} \Omega-\frac{L}{R^{2}} \frac{k^{2} z}{P_{r}} B,
$$

where

$$
\Omega=-\frac{2 H_{v} g k^{2}}{c_{p} P_{r}} \int_{z^{*}=\mathrm{LFC}}^{z^{*}=z} \frac{q_{s, 0}\left(1-\mathrm{RH}_{0}\right)}{T_{0}\left(1+\frac{H_{v}^{2} q_{s, 0}}{c_{p} R_{v} T_{0}^{2}}\right)} d z^{*},
$$

$B_{\mathrm{AD}}$ is the buoyancy for an undiluted air parcel lifted moist adiabatically; $T_{0}, \mathrm{RH}_{0}$, and $q_{s, 0}$ are the virtual temperature, relative humidity, and saturation mixing ratio for liquid water, respectively, of the background environment; $g$ is gravitational acceleration; $H_{v}$ is the latent heat of vaporization; $R$ is the updraft radius; $L$ is a horizontal turbulent mixing length; $P_{r}$ is the turbulent Prandtl number; $k^{2}$ is a constant mixing coefficient; $c_{p}$ is the specific heat of dry air at constant pressure; $R_{v}$ is the gas constant for water vapor; $\Omega$ encapsulates the influences of the entrainment of dry environmental air into the updraft center on $B$; and $-\left(L / R^{2}\right)\left(k^{2} z / P_{r}\right) B$ encapsulates the influences of the entrainment of background environmental temperature on cloud core $B$. This expression is advantageous in that we do not need to assume a vertical profile of fractional entrainment to parameterize the effects of entrainment on $B$. Furthermore, this expression generally agrees well with $B$ in simulated updrafts (e.g., Morrison 2016b, 2017; Peters et al. 2019, 2020a).

Vertically integrating Eq. (4) from $z=0$ to an arbitrary height $z=H$, and using the definition of $w_{B}$ gives

$$
w_{B}^{2}=2 \mathrm{CAPE}_{H}+\frac{2 L}{R^{2}} \int_{z=0}^{z=H} \Omega d z-\frac{2 k^{2} L}{P_{r} R^{2}} \int_{z=0}^{z=H} z B d z,
$$

where $\mathrm{CAPE}_{H} \equiv \int_{z=0}^{z=H} B_{\mathrm{AD}} d z$. Next, we evaluate the third term on the right-hand side of Eq. (6) using integration by parts to obtain

$$
-\frac{2 k^{2} L}{P_{r}} \int_{z=0}^{z=H} z B d z=-\frac{2 k^{2} L H}{P_{r}}\left(\frac{w_{B}^{2}}{2}-\frac{1}{H} \int_{z=0}^{z=H} w_{B}^{2} d z\right),
$$

To approximate the term $(1 / H) \int_{z=0}^{z=H} w_{B}^{2} d z$, we assume a linear profile of $w_{B}$ with height (within this term only) such that $w_{B}(z) \approx(z / H) w_{B}(H)$ giving

$$
-\frac{2 k^{2} L}{P_{r}} \int_{z=0}^{z=H} z B d z=-\frac{2 k^{2} L H}{6 P_{r}} w_{B}^{2}=-\kappa L H w_{B}^{2},
$$

where $\kappa \equiv k^{2} /\left(3 P_{r}\right)$. Combining Eq. (8) with Eq. (6) gives

$$
w_{B}^{2}=2 \mathrm{CAPE}_{H}+\frac{2}{R^{2}} \int_{z=0}^{z=H} \Omega d z-\frac{L H \kappa}{R^{2}} w_{B}^{2} .
$$

Next, make the following definition:

$$
\int_{z=0}^{z=H} \Omega d z=H \bar{\Omega}=-H R K_{H},
$$


where $K_{H} \equiv-\bar{\Omega}$ and the overbar denotes the vertical average of $\Omega$ over depth $H$. Making this substitution, defining the nondimensional updraft radius $\sigma \equiv R / H$, and setting $L=R$ gives $^{3}$

$$
w_{B}^{2}=2 \mathrm{CAPE}_{H}-\frac{2}{\sigma} K_{H}-\frac{\kappa}{\sigma} w_{B}^{2} .
$$

Combining Eq. (11) with Eq. (3) gives

$$
w^{2}=\frac{2 \mathrm{CAPE}_{H}-\frac{2}{\sigma} K_{H}}{1+\frac{\kappa}{\sigma}}-2 \frac{\Delta p_{B}}{\rho_{0}}-2 \frac{\Delta p_{D}}{\rho_{0}} .
$$

Equation (12) now contains four unknown parameters: $w, \sigma$, $\Delta p_{B}$, and $\Delta p_{D}$. To express $\sigma$ in terms of parameters that can be computed from a sounding, we use the azimuthally averaged Boussinesq continuity equation in cylindrical coordinates, which is written as (e.g., Peters et al. 2019)

$$
\frac{\partial r \tilde{u}}{\partial r}+r \frac{\partial \tilde{w}}{\partial z}=0
$$

where the tilde denotes an azimuthal average. Applying $\int_{z=0}^{z=H} \int_{r=0}^{r=R}() d r d z$ to Eq. (13) gives

$$
H R \overline{\widetilde{u_{R}}}+\frac{R^{2}}{2} \hat{w}=0
$$

where $\overline{\widetilde{u_{R}}}$ is the azimuthal and vertical average of $u$ at $R$, and $\hat{w}$ is the area average of $w$ within the updraft at $H$. We assume that the background storm-relative flow $\mathbf{V}_{\mathrm{SR}} \equiv \mathbf{V}_{0}-\mathbf{C}$ (where $\mathbf{C}$ is the storm motion) enters the updraft on the upstream flank at a given level and ascends within the updraft, such that inflow vanishes on the downstream flank of the updraft [this idea is supported by Fig. 12 in Peters et al. (2019)]. At each level, we realign the coordinate system so that $\phi=0$ represents the direction of $\mathbf{V}_{\mathrm{SR}}$. Using these assumptions, we may write

$$
\begin{aligned}
\widetilde{u_{R}} & =\frac{1}{2 \pi} \int_{\phi=-\pi / 2}^{\phi=\pi / 2} \mathbf{V}_{\mathrm{SR}} \cdot \mathbf{r} d \phi \\
& =-\frac{1}{2 \pi} \int_{\phi=-\pi / 2}^{\phi=\pi / 2}\left|\mathbf{V}_{\mathrm{SR}}\right| \cos \phi d \phi=-\frac{\left|\mathbf{V}_{\mathrm{SR}}\right|}{\pi}
\end{aligned}
$$

where $\mathbf{r}$ is the radial direction. Next, we define $V_{\mathrm{SR}} \equiv\left|\mathbf{V}_{\mathrm{SR}}\right|$ and combine Eq. (15) with Eq. (14). In a similar manner to Morrison (2017) and Morrison and Peters (2018), we assume that $\hat{w}$ is proportional to the maximum $w$ at the updraft center, such that $\hat{w} \approx \alpha w$ (where $0<\alpha<1$ is a constant). Using these modifications and approximations, we may rewrite Eq. (14) as

$$
\sigma=\frac{2 \overline{V_{\mathrm{SR}}}}{\pi \alpha w} .
$$

\footnotetext{
${ }^{3}$ In turbulent updrafts with a high Reynolds number, dimensional analysis indicates that $L$ should scale with $R$. This also gives fractional entrainment rates that scale as $1 / R$ consistent with many previous laboratory, theoretical, and modeling studies (Morton et al. 1956; Scorer 1957; Johari 1992; Hernandez-Deckers and Sherwood 2018; Lecoanet and Jeevanjee 2019).
}

This approach for estimating updraft width using the continuity equation produced accurate results when compared to simulations in Peters et al. (2019). Hereinafter, the overbar is omitted on $V_{\mathrm{SR}}$ for simplicity. Combining Eq. (16) with Eq. (12) confines the unknowns to the two pressure terms.

Note that our formula applies for $w$ at any height $H$ within the updraft; however, we wish to make the formula specific to the maximum $w$ within the updraft. Our previous work in Peters (2016) and Morrison and Peters (2018) suggests that while the maximum $w$ typically occurs several kilometers below the equilibrium level (EL), the magnitude of the maximum $w$ corresponds better with buoyant accelerations that are vertically integrated past the height of maximum $w$ to the EL. This is because downward accelerations related to $p_{D}$ near cloud top often displace the maximum $w$ downward from the EL without substantially altering its magnitude. We therefore set $H$ to the EL, replace $\mathrm{CAPE}_{H}$ with CAPE, and $K_{H}$ with $K$, where these quantities are vertically integrated from the level of free convection (LFC) to the EL. Invoking H2, we neglect the pressure terms to obtain the following cubic equation for $w$ :

$$
w^{3}+\frac{2 V_{\mathrm{SR}}}{\kappa \pi \alpha} w^{2}+\frac{2 K}{\kappa} w-\frac{4 V_{\mathrm{SR}}}{\kappa \pi \alpha} \mathrm{CAPE}=0,
$$

which entirely depends on sounding-derived parameters. For instance, CAPE and $K$ are readily evaluated by computing a lifted parcel path and vertically integrating using the soundingderived thermodynamic profile. Furthermore, the constant parameters $\kappa$ and $\alpha$ have commonly used empirical values. At last, $V_{\mathrm{SR}}$ is readily estimated from a vertical wind profile.

A single real and positive solution for $w$ was obtained using the standard cubic formula:

$$
\begin{aligned}
& b=\frac{2 V_{\mathrm{SR}}}{\kappa \pi \alpha}, \quad c=\frac{2 K}{\kappa}, \quad d=-\frac{4 V_{\mathrm{SR}}}{\kappa \pi \alpha} \mathrm{CAPE}, \\
& \psi_{1}=b^{2}-3 c, \quad \psi_{2}=2 b^{3}-9 b c+27 d, \quad \text { and } \\
& w_{t, B}=-\frac{1}{3}\left\{b+\left[\frac{\psi_{2}+\left(\psi_{2}^{2}-4 \psi_{1}^{3}\right)^{1 / 2}}{2}\right]^{1 / 3}\right. \\
& \left.+\psi_{1}\left[\frac{2}{\psi_{2}+\left(\psi_{2}^{2}-4 \psi_{1}^{3}\right)^{1 / 2}}\right]^{1 / 3}\right\},
\end{aligned}
$$

where $w_{t, B}$ henceforth refers to theoretical (hence the subscript " $t$ ") solutions to Eq. (18) that incorporates buoyancy accelerations only (hence the subscript " $B$ "). These solutions are analogous to estimating maximum updraft $w$ using ECAPE [i.e., $w \approx(2 \mathrm{ECAPE})^{1 / 2}$ ] but have the advantage of not requiring a prespecified fractional entrainment rate. Rather, entrainment rates, and the associated influence of entrainment on buoyancy, are predicted through the inclusion of the sounding-derived parameters $K$, and $V_{\mathrm{SR}}$, and the constant parameters $\kappa$ and $\alpha$ have commonly used empirical values. For a complete assessment of $\mathrm{H} 1$, we must also obtain a theoretical expression for $B$ (hereinafter $B_{t}$ ) to demonstrate that entrainment-driven variations in $B$ are responsible for the behavior of $w_{t, B}$. To accomplish this, we used $w_{t, B}$ to evaluate 


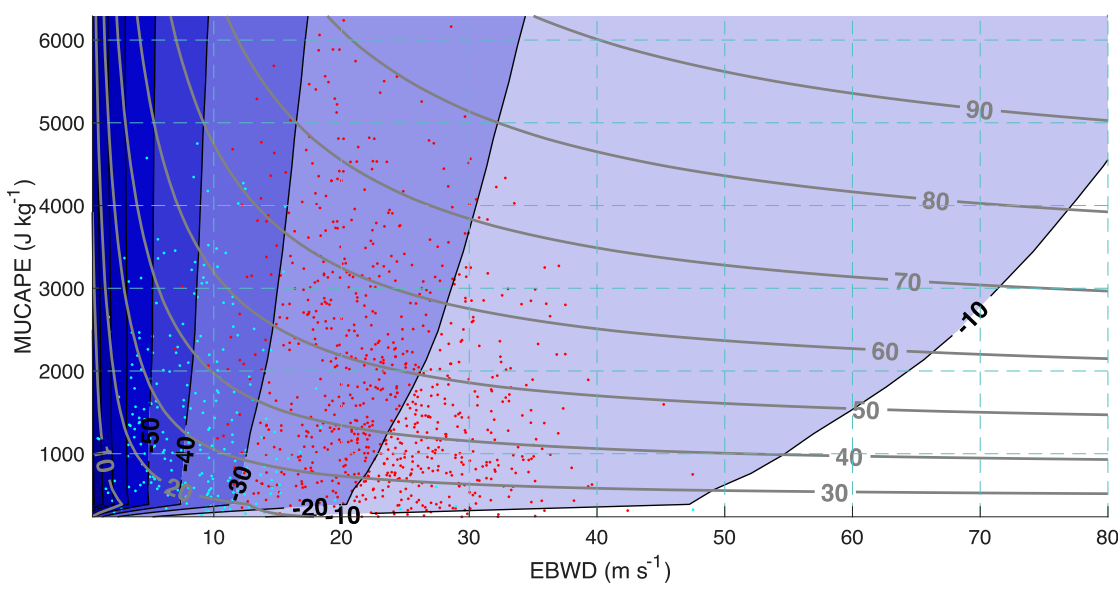

FIG. 1. Solutions for $w_{t, B}$ from Eq. (18) (gray contours; $\mathrm{m} \mathrm{s}^{-1}$ ), and the quantity $\left[(2 \mathrm{MUCAPE})^{1 / 2}-w_{t, B}\right] /(2 \mathrm{MUCAPE})^{1 / 2}(\%$; shading) which measures the percentage difference of the theoretical solution from (2MUCAPE) $)^{1 / 2}$. Solutions are plotted as a function of the EBWD ( $x$ axis; $\mathrm{m} \mathrm{s}^{-1}$ ) and the MUCAPE from the Weisman and Klemp (1982) sounding $\left(y\right.$ axis; $\mathrm{J} \mathrm{kg}^{-1}$ ). Values of EBWD and MUCAPE from T03 supercell soundings are shown as red dots, and nonsupercell soundings are shown as cyan dots.

Eq. (16), combined this equation with Eq. (4), and rearranged using various definitions used in this section to obtain

$$
B_{t}(z)=\frac{B_{\mathrm{AD}}(z)+\left(\frac{\pi \alpha w_{t, B}}{2 V_{S R}}\right)^{2} \frac{\Omega(z)}{H}}{1+\left(\frac{\pi \alpha w_{t, B}}{2 V_{S R}}\right)^{2} \frac{3 \kappa z}{H}} .
$$

Here, we assume that $\sigma$ is constant through the depth of the updraft and set $H$ to the height of maximum $B$, which is roughly consistent with where the height of maximum $w$ occurs in the simulations by Peters (2016). Also note that, unlike $w_{t, B}, B_{t}$ is a function of height.

\section{Behavior of the solution for $w$ in the parameter space of CAPE and vertical wind shear}

To illustrate the general behavior of the formula for $w_{t, B}$, we applied it to the analytic thermodynamic sounding of Weisman and Klemp (1982) with the relative humidity (RH) above $3 \mathrm{~km}$ set to a uniform value of $45 \%$. To evaluate a range of CAPE values, boundary layer mixing ratios from 9.5 through $18 \mathrm{~g} \mathrm{~kg}^{-1}$ were evaluated at intervals of $0.5 \mathrm{~g} \mathrm{~kg}^{-1}$. The range of most unstable CAPE (MUCAPE) values for this boundary layer mixing ratio range was roughly $500-6300 \mathrm{~J} \mathrm{~kg}^{-1}$. For the sake of simplicity, we used the MUCAPE in the formula for $w_{t, B}$, although it is not obvious that this is the best choice for all situations. A comprehensive evaluation of $w_{t, B}$ using alternative formulas for CAPE, however, is left to future work.

The formula also requires a specification of $V_{\mathrm{SR}}$. In principle, one could use the storm motion estimate of Bunkers et al. (2000) to compute $V_{\mathrm{SR}}$ from a given wind profile. However, not all deep moist convection (including supercells) moves in accordance with this motion estimate. In particular, cumulonimbus updrafts in weaker shear tend to be composed of a series of rising thermals, rather than continuous steady plume- like updrafts (e.g., Sherwood et al. 2013; Romps and Charn 2015; Hernandez-Deckers and Sherwood 2016; Morrison et al. 2020; Peters et al. 2020a). It is unclear whether the motion of the cloud as a whole or the individual thermals are most relevant in these situations. To keep the estimate of $V_{\mathrm{SR}}$ general, we simply set this value to half of the effective bulk wind difference (EBWD; defined in Thompson et al. 2007). ${ }^{4}$ EBWD values ranging from 0 to $80 \mathrm{~m} \mathrm{~s}^{-1}$ were used to evaluate Eq. (18). Constant values of $k^{2}=0.2$ (consistent with Morrison 2017) and $\alpha=0.8$ (consistent with Morrison and Peters 2018) were also used for all subsequent calculations. Last, MUCAPE and EBWD values from the Thompson et al. (2003) proximity sounding database (hereinafter the T03 soundings) were compared with the theoretical solutions to show the range of these values typically encompassed by observed severe weather environments.

For a fixed value of EBWD, $w_{t, B}$ generally increases with increasing MUCAPE (Fig. 1). This connection is intuitive because MUCAPE should at least partially determine updraft $B$ and thus $w_{t, B}$. Likewise, $w_{t, B}$ generally increases with increasing EBWD for a fixed MUCAPE value because updrafts become wider and entrainment dilutes their core buoyancy to a lesser extent when the EBWD is large (Fig. 1). This solution behavior corroborates our recent work in Peters et al. (2019) which

\footnotetext{
${ }^{4}$ This assumes that storm motion is entirely advective (i.e., no propagation). Analogous calculations to the ones shown here with the storm motion estimate of Bunkers et al. (2000) agreed with this paper's results for large wind shear values but deviated somewhat from this paper's results at small wind shear values. This discrepancy is because the Bunkers et al. (2000) estimate assumes a constant propagation speed of $7.5 \mathrm{~m} \mathrm{~s}^{-1}$, which gives an unrealistically high lower bound for $V_{\mathrm{SR}}$ and likely overestimates propagation speeds in weakly sheared environments.
} 
showed that similar updraft speeds are possible in low-CAPE, high-shear environments and in high-CAPE, low-shear environments (note that the $w_{t, B}$ contours vertically asymptote on the left side of the figure and then horizontally near the right side of Fig. 1). We also assessed the sensitivity of this solution to the parameters $k^{2}$ and $\alpha$ (not shown). Changes to these parameters that were on the order of $50 \%$ yielded maximum differences in $w_{t, B}$ of $15 \%$ in the region encompassed by T03 supercell soundings, and in most cases these differences were much less than $15 \%$. Furthermore, these parameter changes did not appreciably change the shape of the solution within the parameter space shown in Fig. 1. This suggests that the formula for $w_{t, B}$ is relatively insensitive to the choices for these parameters.

To close this section, we briefly examine how $w_{t, B}$ compares to MUCAPE in the T03 soundings. The quantity $(1 / 2) w_{t, B}^{2}$ has the same units as CAPE and is therefore a closer analogy to CAPE than $w_{t, B}$. A comparison of $(1 / 2) w_{t, B}^{2}$ and MUCAPE among the T03 soundings (Fig. 2) shows $2000-3000 \mathrm{~J} \mathrm{~kg}^{-1}$ of spread in MUCAPE for a given $(1 / 2) w_{t, B}^{2}$ value and $1500 \mathrm{~J} \mathrm{~kg}^{-1}$ of spread in $(1 / 2) w_{t, B}^{2}$ for a given MUCAPE value. The EBWD magnitude is predominantly responsible for this spread, with MUCAPE much closer to $(1 / 2) w_{t, B}^{2}$ (i.e., closer to the 1-to-1 line) when the EBWD is large than when the EBWD is small (i.e., farther to the upper left of the 1-to-1 line). Based on this behavior, we generally expect $w_{t, B}$ to give close estimates to MUCAPE for maximum updraft $w$ when shear is large, but much smaller estimates for maximum updraft $w$ than MUCAPE when shear is small.

\section{Comparisons of theoretical formulas with simulated supercells}

To quantitatively evaluate our hypotheses, we analyzed the 54 idealized simulations of Peters et al. (2019), the 46 idealized simulations of Peters et al. (2020b), and the 15 idealized simulations of Nowotarski et al. (2020). These collections of simulations are referred to as the P19, P20, and N20 runs, respectively. To evaluate $\mathrm{H} 1$, we compare $w_{t, B}$ with maximum $w$ within simulated updrafts, along with other theoretical quantities such as MUCAPE and ECAPE (see the appendix) that are commonly used to assess maximum $w$ in updrafts. Support for $\mathrm{H} 1$ would involve more skillful predictions of maximum updraft $w$ by $w_{t, B}$ than from MUCAPE and ECAPE. To evaluate $\mathrm{H} 2$, we compare integrated DPA to integrated BPA along trajectories from simulations. Support for $\mathrm{H} 2$ would involve nearly equal and offsetting integrated DPA and $\mathrm{BPA}$ in simulations, resulting in little contribution by net pressure forces to maximum $w$.

All simulations share a similar modeling configuration, using Cloud Model 1 (CM1; Bryan and Fritsch 2002) version 18 with a 100 by $100 \mathrm{~km}$ horizontal domain extent and horizontal grid spacing of $250 \mathrm{~m}$. Vertical domain dimensions were 18, 20, and $20 \mathrm{~km}$ for the P19, P20, and N20 runs, respectively. Vertical grid spacing was 100,100 , and stretched from 50 to $250 \mathrm{~m}$ between 2 and $7 \mathrm{~km}$ in the P19, P20, and N20 runs, respectively. The P19 and P20 runs used the double-moment microphysics scheme of Morrison et al. (2009), whereas the N20 runs used the NSSL double-moment microphysics scheme. Convection

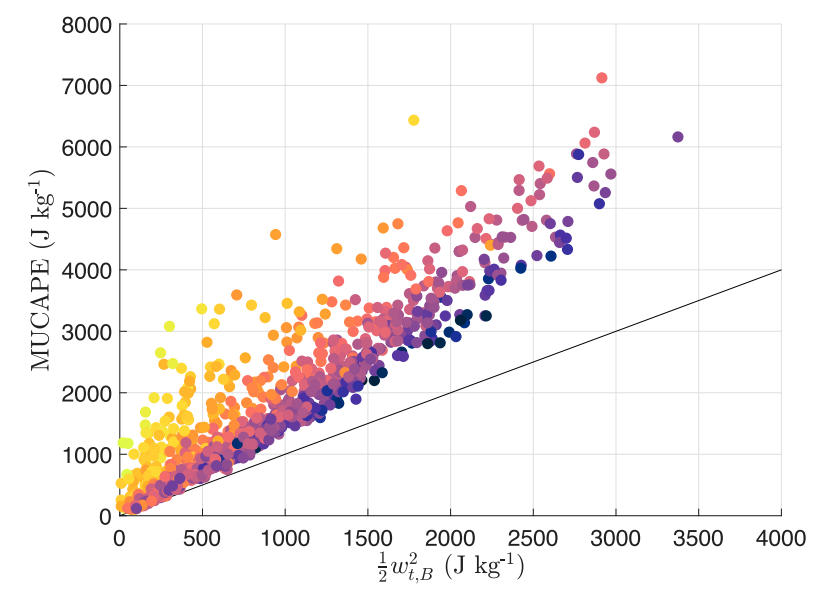

FIG. 2. Plot of $(1 / 2) w_{t, B}^{2}\left(\mathrm{~J} \mathrm{~kg}^{-1} ; x\right.$ axis $)$ vs MUCAPE $\left(\mathrm{J} \mathrm{kg}^{-1}\right.$; $y$ axis) from the T03 proximity soundings. The 1-to- 1 line is shown in black. The color of the dots shows the relative magnitude of the EBWD, with dark purple indicating large EBWD magnitudes, and yellow indicating small EBWD magnitudes.

in the P19 and P20 simulations was initiated with a warm bubble, whereas the updraft forcing method of Naylor and Gilmore (2012) was used in the N20 simulations. Initial model environments featured a range of MUCAPE, vertical wind shear, and middle tropospheric $\mathrm{RH}$ values which resulted in a range of convective modes from multicelled clusters to sustained supercell updrafts. Wind and thermodynamic profiles were analytically generated in the P19 and P20 runs and on the basis of proximity soundings to severe weather environments in the N20 runs. Initial conditions in these simulations were horizontally homogeneous (aside from the features included to initiate convection). Other attributes of these simulations are available in Peters et al. (2019, 2020b) and Nowotarski et al. (2020).

Our theoretical calculations of MUCAPE, $K$, and RH came from the initial model sounding for each simulation. These solutions were compared with 1-h averages of the instantaneous domain maximum $w$ during the 2-3 h-time range in the $\mathrm{P} 19$ and $\mathrm{P} 20$ runs and the $1-2 \mathrm{~h}$ time range in the N20 runs (hereinafter simply "simulated w"). Simulated $w$ was also compared with (2MUCAPE) ${ }^{1 / 2}$ and $(2 \mathrm{ECAPE})^{1 / 2}$. To compute ECAPE, we used fractional entrainment rates $\varepsilon$ of $3 \times 10^{-3}$ and $1 \times 10^{-3} \mathrm{~m}^{-1}$ (the former value was used in previous studies of tropical convection; e.g., Zhang 2009).

Our comparison between theory and the simulations uses the following integrated accelerations along back trajectories in the simulations:

$$
\begin{aligned}
w_{B} & \equiv \max \left(\sqrt{2 \int_{z^{*}=z_{0}}^{z^{*}=z} B d z^{*}}\right), \\
w_{\mathrm{BPA}} & \equiv-\max \left[-i \sqrt{2 \int_{z^{*}=z_{0}}^{z^{*}=z}-\left(1 / \rho_{0}\right)\left(\partial p_{B} / \partial z\right) d z^{*}}\right],
\end{aligned}
$$



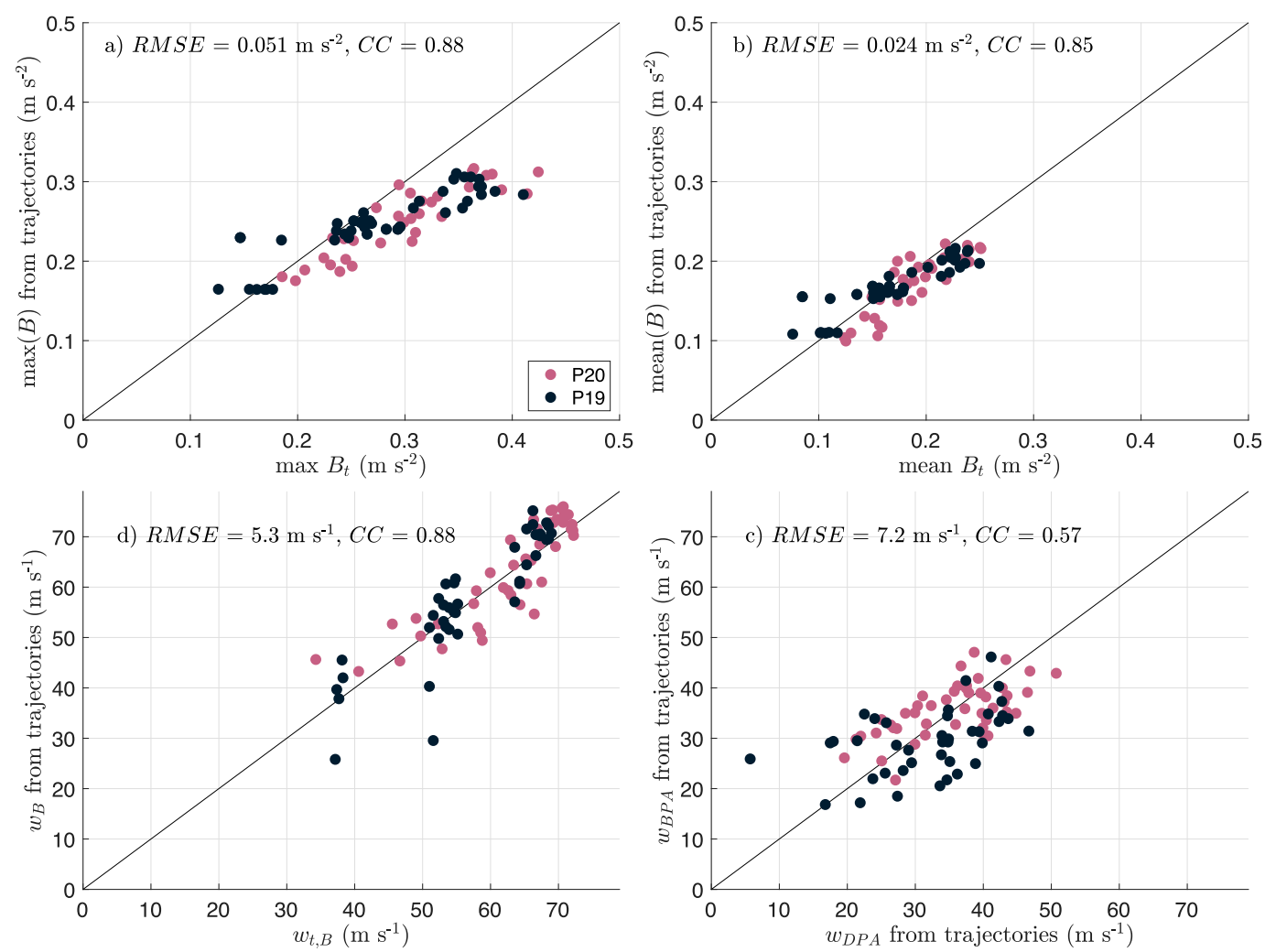

FIG. 3. (a) Maximum $B_{t}\left(x\right.$ axis; $\left.\mathrm{m} \mathrm{s}^{-2}\right)$ vs maximum $B$ ( $y$ axis; $\left.\mathrm{m} \mathrm{s}^{-2}\right)$ from trajectories in the $\mathrm{P} 20$ and $\mathrm{P} 19$ simulations. (b) Mean $B_{t}\left(x\right.$ axis; $\left.\mathrm{m} \mathrm{s}^{-2}\right)$ vs mean $B\left(y\right.$ axis; $\left.\mathrm{m} \mathrm{s}^{-2}\right)$ from trajectories (means are computed over the vertical extent of $B>0)$. (c) The $w_{t, B}\left(x\right.$ axis; $\left.\mathrm{m} \mathrm{s}^{-1}\right)$ vs $w_{B}\left(y\right.$ axis; $\left.\mathrm{m} \mathrm{s}^{-1}\right)$ from trajectories in the $\mathrm{P} 20$ and P19 simulations. (d) The $w_{\text {DPA }}\left(x\right.$ axis; $\left.\mathrm{m} \mathrm{s}^{-1}\right)$ vs $w_{\mathrm{BPA}}\left(y\right.$ axis; $\left.\mathrm{m} \mathrm{s}^{-1}\right)$ from trajectories in the P20 and P19 simulations. In all panels, the P20 and P19 runs are purple and black dots, respectively. One-to-one lines are shown in black. Root-mean-square errors (RMSEs) and linear Pearson correlation coefficients (CC) are included in the figures.

$$
w_{\mathrm{NET}} \equiv \max \left\{\sqrt{2 \int_{z^{*}=z_{0}}^{z^{*}=z}\left[B-\left(1 / \rho_{0}\right)\left(\partial p_{B} / \partial z\right)-\left(1 / \rho_{0}\right)\left(\partial p_{D} / \partial z\right)\right] d z^{*}}\right\}, \quad \text { and }
$$

$$
w_{\mathrm{DPA}} \equiv \max \left\{\sqrt{2 \int_{z^{*}=z_{0}}^{z^{*}=z}\left[-\left(1 / \rho_{0}\right)\left(\partial p_{D} / \partial z\right)\right] d z^{*}}\right\}
$$

where $z_{0}$ is initial trajectory height, max is the maximum over all $z$, and the $i \equiv(-1)^{1 / 2}$ is included in the formula for $w_{\mathrm{BPA}}$ to ensure that a negative real value is returned by the formula. All back trajectories were released from the point of maximum domain $w$ at a given time. Note that $B$ calculations from all simulations include the influence of precipitation loading, which is neglected in $w_{t, B}$. The potential implications of this omission in $w_{t, B}$ are discussed later in this section. In subsequent analysis, we used the average over roughly 50 trajectories per simulation. Further information on the computation of these quantities is available in P19 and P20 (no back trajectories were computed in N20).

As a first step toward addressing $\mathrm{H} 1$, we compare $B_{t}$ with $B$ along trajectories from the simulations. Maximum (Fig. 3a) and mean (Fig. 3b) $B_{t}$ values are well correlated with the comparable quantities along trajectories ( $\mathrm{CC}=0.88$ and 0.85 , respectively, where $\mathrm{CC}$ is a linear Pearson correlation coefficient), suggesting that our entrainment formulation adequately emulates entrainment in the simulations. Furthermore, root-mean-square errors (RMSEs) are only a small fraction of the $B$ magnitudes. Note that there is a slight bias toward overprediction of $B$ for both maximum and mean values (points more frequently fall to the right of the one-to-one line in Figs. $3 a$ and $3 b$ ), which is a potential consequence of neglecting precipitation loading in the formulation of $B_{t}$. Similarly, $w_{t, B}$ and $w_{B}$ are strongly correlated (Fig. $3 \mathrm{c} ; \mathrm{CC}=$ 0.88 ) with an RMSE of $5.3 \mathrm{~m} \mathrm{~s}^{-1}$, which is on the order of $10 \%$ of the typical magnitudes of $w_{B}$. These results suggest that our theoretical treatment of the influence of entrainment on buoyancy is consistent with what occurs in the simulations.

In support of $\mathrm{H} 2$, we find a general trend of offset between BPA and DPA was present in the P19 and P20 simulations 
(Fig. 3d), as illustrated by a moderate linear Pearson correlation coefficient $\mathrm{CC}=0.57$ between $w_{\mathrm{DPA}}$ and $w_{\mathrm{BPA}}$ along with an RMSE of $7.2 \mathrm{~m} \mathrm{~s}^{-1}$. Given that magnitudes of $w_{\mathrm{BPA}}$ and $w_{\mathrm{DPA}}$ were generally on the order of $30-50 \mathrm{~m} \mathrm{~s}^{-1}$, the aforementioned RMSE value suggests substantial offset between these two forces.

Finally, we compare solutions for $w_{t, B}$ to domain maximum $w$ in the N20 runs, and to $w_{\mathrm{NET}}$ along simulated trajectories in the P19 and P20 runs. Solutions for $w_{t, B}$ show an RMSE of $8 \mathrm{~m} \mathrm{~s}^{-1}$ (Fig. 4a), which is less than half of the RMSE for $(2 \mathrm{MUCAPE})^{1 / 2}$ and (2ECAPE) $)^{1 / 2}$ with $\varepsilon=3 \times 10^{-3}$, and slightly less than $\sqrt{2 \mathrm{ECAPE}}$ with $\varepsilon=1 \times 10^{-3}$ (though the later difference was not statistically significant). The utility of $w_{t, B}$ over all the alternative measures for $w$ tested here is emphasized when these formulas are applied to the P19 and P20 runs (Fig. 4b). These simulations were only run with three different values of MUCAPE, so the predictive expressions for updraft strength that depend on thermodynamics only are extremely limited in their ability to discriminate differences in updraft intensity among the runs. Accordingly, the RMSE of our theoretical expression ranged from about one-half to onefifth of the other updraft strength measures in the P19 and P20 simulations.

\section{Summary, conclusions, and discussion}

This article leverages recent improvements in our understanding of supercell dynamics to derive a theoretical expression for the maximum $w$ in supercell thunderstorms from the vertical momentum equation, a thermodynamic equation, and the continuity equation. In constructing this expression, we hypothesized that fractional entrainment rates are dependent on the magnitude of the environmental vertical wind shear (H1) and used this hypothesized connection to predict the influence of entrainment on updraft buoyancy. We also hypothesized that the net influence of perturbation pressure effects on maximum $w$ in supercell thunderstorms is negligible (H2) and therefore neglected these effects in our formulation for maximum $w$. To validate these hypotheses, along with the general ability of our theoretical expression to predict maximum $w$ in supercells, the results of our formula were compared to the output from recent high-resolution numerical simulations.

General conclusions from our results are as follows:

- Our theoretical framework accurately predicts updraft $B$, which lends credence to the treatment of entrainment in our expression.

- Our theoretical expression for maximum $w$ substantially improves predictions of trajectory maximum $w$ in simulated updrafts over existing diagnostic measures for updraft intensity, such as MUCAPE and ECAPE, further supporting H1.

- Integrated pressure accelerations along back trajectories in numerical simulations show large offsetting contributions from DPA and BPA resulting in very small net pressure influences on updraft maximum $w$, which supports $\mathrm{H} 2$.

The results of this article emphasize the importance of entrainment in supercell environments, and $w_{t, B}$ is advantageous over existing measures of ECAPE in that we do not need to specify a fractional entrainment rate for the computation. Rather, the formula uses dynamical connections between
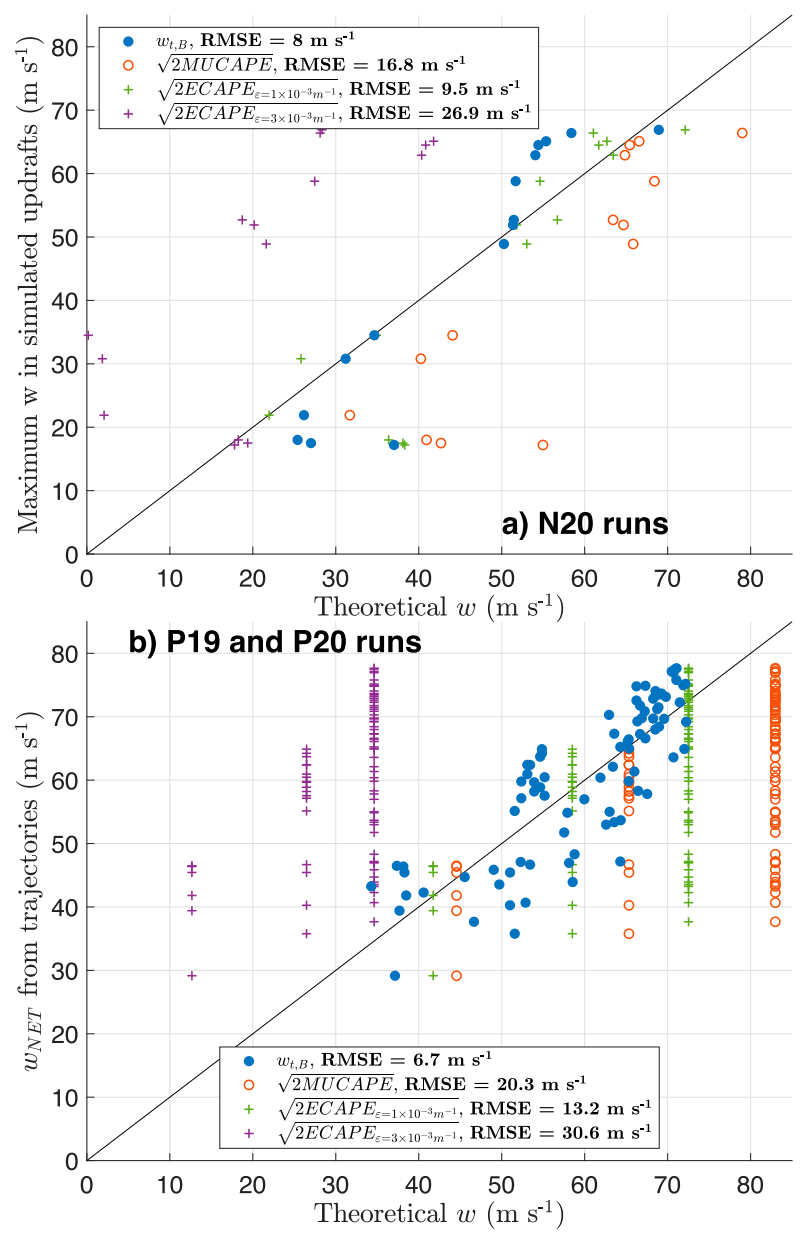

FIG. 4. A comparison of (a) different theoretical $w$ values ( $x$ axis; $\mathrm{m} \mathrm{s}^{-1}$ ) vs 1-h averages of the instantaneous maximum in $w$ ( $y$ axis; $\left.\mathrm{m} \mathrm{s}^{-1}\right)$ in the $\mathrm{N} 20$ runs and (b) theoretical $w$ values $\left(x\right.$ axis; $\left.\mathrm{m} \mathrm{s}^{-1}\right)$ vs the average over all $w_{\text {NET }}\left(y\right.$ axis; $\mathrm{m} \mathrm{s}^{-1}$ ) along trajectories in the P19 and P20 simulations. Solutions for $w_{t, B}$ (blue dots), for ECAPE with $\varepsilon=1 \times 10^{-3}$ (green plus signs), ECAPE with $\varepsilon=3 \times 10^{-3}$ (purple plus signs), and MUCAPE (red open circles). One-to-one lines are shown in black. RMSEs are shown in the legends. The 1-2-h average of the domain maximum $w$ was used from the N20 simulations.

vertical wind shear and updraft width to predict the effects of entrainment on buoyancy. We must emphasize that $w_{t, B}$ offers a prediction of the updraft maximum $w$, rather than a representation of the vertical variations in this quantity. Accordingly, this quantity is most relevant to the forecasting and research of processes that are strongly connected to the updraft maximum $w$, such as electrification, hail production, downdraft strength, and the discrimination between supercellular and nonsupercellular storm modes.

A comprehensive assessment of the utility of this parameter in forecasting is beyond the scope of this study; however, the parameter's potential utility is illustrated by a simple demonstration. In Peters et al. (2020b), we used the true skill statistic (TSS) to assess the ability of various thermodynamic and wind related parameters computed from the T03 proximity sounding 
database to discriminate supercell versus nonsupercellular storm modes. TSS ranges from 0 to 1 , with 1 indicating perfect performance and 0 indicating no skill. In that study, all parameters were normalized by their standard deviations and the discriminating threshold for each parameter was simply set to the normalized value that yielded the largest TSS [more details are available in Peters et al. (2020b)]. Using the same strategy here, we find that the TSSs for $0-1-\mathrm{km}$ mean CAPE and MUCAPE are 0.21 and 0.14 , respectively, suggesting that CAPE is a poor discriminator between supercells and nonsupercells (consistent with T03). The TSS for $w_{t, B}$, on the other hand, is 0.45 . The TSS for the supercell composite parameter (SCP; Thompson et al.2007), which is defined as the effective storm-relative helicity times the MUCAPE times the EBWD divided by a scale factor, is 0.68 . Replacing MUCAPE with $w_{t, B}$ in the SCP increases the TSS to $0.79-\mathrm{a} 17 \%$ improvement. All of these differences were found to be statistically significant using a Student's $t$ test.

While it is quite possible that the updraft maximum $w$ and low-level (i.e., below $2 \mathrm{~km}$ ) $w$ are correlated, there are extraneous factors influencing low-level $w$ such as low-level dynamic accelerations related to a storm's mesocyclone, which have not been accounted for here. The applications of this work to tornado forecasts and research are therefore unclear and require further investigation. One last potential application of $w_{t, B}$ is in the forecasting of convection initiation. On days on which CAPE is large but low midlevel RH prevents the development of deep convection, $w_{t, B}$ may better reflect the large impact of entrainment on updraft intensity relative to existing measures of CAPE.

Acknowledgments. We are grateful for the thoughtful comments from Dr. Louis Wicker and three anonymous peer reviewers. Authors J. Peters's and C. Nowotarski's efforts were supported by National Science Foundation (NSF) Grants AGS-1928666 and AGS-1928319, respectively. In addition, Peters was partially supported by NSF Grants AGS-1841674 and Department of Energy Atmospheric System Research (DOE ASR) Grant DE-SC0000246356. Author H. Morrison was partially supported by DOE ASR Grant DE-SC0020104.

Data availability statement. Please reference Peters et al. (2019, 2020b) and Nowotarski et al. (2020) for the model data used in this study.

\section{APPENDIX}

\section{Entrainment CAPE}

The equation for moist static energy $h$ may be written as

$$
h=c_{p} T+H_{v} q_{v}+g z,
$$

where $q_{v}$ is the water vapor mixing ratio. If we define $h_{0}(z)$ as a height-dependent background profile and $h^{\prime} \equiv h-h_{0}$, it follows that

$$
h^{\prime}=c_{p} T^{\prime}+H_{v} q_{v}^{\prime}
$$

Next, we multiply by $g /\left(c_{p} T_{0}\right)$ and assume that an updraft core is saturated such that $q_{v}^{\prime}=q_{s}-q_{0}$. At this point, one may solve for $T^{\prime}$ and $q_{s}$ (if the profile of $h^{\prime}$ is known) by using a numeric root solver and the Clausius-Clapeyron relation. However, for simplicity here we assume that $q_{s} \approx q_{s, 0}$ to yield a comparatively simple analytic expression. Applying the above assumptions and rearranging yields

$$
B=\frac{g}{c_{p} T_{0}} h^{\prime}-\frac{g H_{v} q_{s, 0}}{c_{p} T_{0}}\left(1-\mathrm{RH}_{0}\right),
$$

where $B \approx g\left(T_{v} / T_{v, 0}\right) \approx g\left(T / T_{0}\right)$. Note that vertical profiles of $\mathrm{RH}_{0}, T_{0}$, and $q_{s, 0}$ are obtainable from a given atmospheric sounding. Vertically integrating from $z=\operatorname{LFC}$ to $z=\mathrm{EL}$ gives

$$
\mathrm{ECAPE}=\frac{g}{c_{p} T_{0}} \int_{z=\mathrm{LFC}}^{z=\mathrm{EL}} h^{\prime} d z-\frac{g H_{v}}{c_{p}} \int_{z=\mathrm{LFC}}^{z=\mathrm{EL}} \frac{q_{s, 0}}{T_{0}}\left(1-\mathrm{RH}_{0}\right) d z .
$$

The plume equation for a conserved variable $C$ is commonly written as $\partial C / \partial z=\partial C^{\prime} / \partial z-\partial C_{0} / \partial z=-\varepsilon\left(C-C_{0}\right)=-\varepsilon C^{\prime}$, where $\varepsilon$ is a fractional entrainment length scale. Assuming that the quantity $h$ is conserved following an ascending air parcel, we may use this equation to write

$$
\frac{\partial h^{\prime}}{\partial z}=\frac{\partial h_{0}}{\partial z}-\varepsilon h^{\prime}
$$

The particular solution to this equation with $h^{\prime}=0$ at the LFC is

$$
h^{\prime}(z)=e^{\varepsilon z} \int_{z^{*}=\mathrm{LFC}}^{z^{*}=z} e^{-\varepsilon z^{*}} \frac{d h_{0}}{d z} d z^{*} .
$$

Combining Eq. (A6) with Eq. (A4) gives

$$
\begin{aligned}
\mathrm{ECAPE}= & \frac{g}{c_{p} T_{0}} \int_{z=\mathrm{LFC}}^{z=\mathrm{EL}}\left(e^{\varepsilon z} \int_{z^{*}=\mathrm{LFC}}^{z^{*}=z} e^{-\varepsilon z^{*}} \frac{d h_{0}}{d z^{*}} d z^{*}\right) d z \\
& -\frac{g H_{v}}{c_{p}} \int_{z=\mathrm{LFC}}^{z=\mathrm{EL}} \frac{q_{s, 0}}{T_{0}}\left(1-\mathrm{RH}_{0}\right) d z,
\end{aligned}
$$

where all quantities on the right-hand side are obtainable from an environmental sounding, given a prespecified fractional entrainment length scale. To evaluate the efficacy of the two major assumptions in this derivation $\left(g\left(T_{v} / T_{v, 0}\right) \approx g\left(T / T_{0}\right)\right.$ and $\left.q_{s} \approx q_{s, 0}\right)$, the results of Eq. (A7) with $\varepsilon$ set to 0 were compared with standard CAPE calculations, yielding reasonable agreement (not shown). Note that in this case the EL and LFC are defined as the heights for which $h^{\prime}$ initially and last is equal to zero, respectively.

\section{REFERENCES}

Ahmed, F., and J. D. Neelin, 2018: Reverse engineering the tropical precipitation-buoyancy relationship. J. Atmos. Sci., 75, 1587-1608, https://doi.org/10.1175/JAS-D-17-0333.1.

Arakawa, A., and W. H. Schubert, 1974: Interaction of a cumulus cloud ensemble with the large-scale environment, part I. J. Atmos. Sci., 31, 674-701, https://doi.org/10.1175/15200469(1974)031<0674:IOACCE>2.0.CO;2.

Browning, K. A., 1963: The growth of large hail within a steady updraught. Quart. J. Roy. Meteor. Soc., 89, 490-506, https:// doi.org/10.1002/qj.49708938206.

Bryan, G. H., and J. M. Fritsch, 2002: A benchmark simulation for moist nonhydrostatic numerical models. Mon. Wea. Rev., 130, 
2917-2928, https://doi.org/10.1175/1520-0493(2002)130<2917: ABSFMN $>2.0 . \mathrm{CO} ; 2$.

Bunkers, M. J., B. A. Klimowski, R. L. Thompson, and M. L. Weisman, 2000: Predicting supercell motion using a new hodograph technique. Wea. Forecasting, 15, 61-79, https://doi.org/ 10.1175/1520-0434(2000)015<0061:PSMUAN>2.0.CO;2.

Coffer, B. E., and M. D. Parker, 2015: Impacts of increasing low-level shear on supercells during the early evening transition. Mon. Wea. Rev., 143, 1945-1969, https://doi.org/10.1175/MWR-D-14-00328.1.

Cotton, W. R., 1975: On parameterization of turbulent transport in cumulus clouds. J. Atmos. Sci., 32, 548-564, https://doi.org/ 10.1175/1520-0469(1975)032<0548:OPOTTI >2.0.CO;2.

Davies-Jones, R., 2002: Linear and nonlinear propagation of supercell storms. J. Atmos. Sci., 59, 3178-3205, https://doi.org/ 10.1175/1520-0469(2003)059<3178:LANPOS $>2.0$.CO 2 .

_ 2003: An expression for effective buoyancy in surroundings with horizontal density gradients. J. Atmos. Sci., 60, 2922-2925, https://doi.org/10.1175/1520-0469(2003)060<2922:AEFEBI> 2.0.CO;2.

De Roode, S. R., A. P. Siebesma, H. J. Jonker, and Y. De Voog, 2012: Parameterization of the vertical velocity equation for shallow cumulus clouds. Mon. Wea. Rev., 140, 2424-2436, https://doi.org/10.1175/MWR-D-11-00277.1.

Doswell, C. A., III, and P. M. Markowski, 2004: Is buoyancy a relative quantity? Mon. Wea. Rev., 132, 853-863, https://doi.org/ 10.1175/1520-0493(2004)132<0853:IBARQ>2.0.CO;2.

——, H. E. Brooks, and R. A. Maddox, 1996: Flash flood forecasting: An ingredients-based methodology. Wea. Forecasting, 11, 560-581, https://doi.org/10.1175/1520-0434(1996) $011<0560$ :FFFAIB $>2.0$. CO;2.

Emanuel, K. A., 1994: Atmospheric Convection. Oxford University Press, 588 pp.

Gallus, W. A. J., N. A. Snook, and E. V. Johnson, 2008: Spring and summer severe weather reports over the Midwest as a function of convective mode: A preliminary study. Wea. Forecasting, 23, 101-113, https://doi.org/10.1175/2007WAF2006120.1.

Hannah, W. M., 2017: Entrainment versus dilution in tropical deep convection. J. Atmos. Sci., 74, 3725-3747, https://doi.org/ 10.1175/JAS-D-16-0169.1.

Hernandez-Deckers, D., and S. C. Sherwood, 2016: A numerical investigation of cumulus thermals. J. Atmos. Sci., 73, 4117-4136, https://doi.org/10.1175/JAS-D-15-0385.1.

— cumulus thermals. J. Atmos. Sci., 75, 3911-3924, https:// doi.org/10.1175/JAS-D-18-0077.1.

Holloway, C. E., and J. D. Neelin, 2009: Moisture vertical structure, column water vapor, and tropical deep convection. J. Atmos. Sci., 66, 1665-1683, https://doi.org/10.1175/2008JAS2806.1.

Johari, H., 1992: Mixing in thermals with and without buoyancy reversal. J. Atmos. Sci., 49, 1412-1426, https://doi.org/10.1175/ 1520-0469(1992)049<1412:MITWAW>2.0.CO;2.

Kyle, T. G., W. R. Sand, and D. J. Musil, 1976: Fitting measurements of thunderstorm updraft properties to model profiles. Mon. Wea. Rev., 104, 611-617, https://doi.org/10.1175/15200493(1976)104<0611:FMOTUP>2.0.CO;2.

Lecoanet, D., and N. Jeevanjee, 2019: Entrainment in resolved, dry thermals. J. Atmos. Sci., 76, 3785-3801, https://doi.org/10.1175/ JAS-D-18-0320.1.

Mapes, B. E., and R. Neale, 2011: Parameterizing convective organization to escape the entrainment dilemma. J. Adv. Model. Earth Sys., 3, M06004, https://doi.org/10.1029/2011ms000042.

Marion, G. R., and R. Trapp, 2019: The dynamical coupling of convective updrafts, downdrafts, and cold pools in simulated supercell thunderstorms. J. Geophys. Res. Atmos, 124, 664-683, https://doi.org/10.1029/2018JD029055.

McCaul, E. W., and M. L. Weisman, 1996: Simulations of shallow supercell storms in landfalling hurricane environments. Mon. Wea. Rev., 124, 408-429, https://doi.org/10.1175/15200493(1996)124<0408:SOSSSI >2.0.CO;2.

Moncrieff, M. W., and M. J. Miller, 1976: The dynamics and simulation of tropical cumulonimbus and squall lines. Quart. J. Roy. Meteor. Soc., 102, 373-394, https://doi.org/10.1002/qj.49710243208.

Morrison, H., 2016a: Impacts of updraft size and dimensionality on the perturbation pressure and vertical velocity in cumulus convection. Part I: Simple, generalized analytic solutions. J. Atmos. Sci., 73, 1441-1454, https://doi.org/10.1175/JAS-D-15-0040.1.

_ 2016b: Impacts of updraft size and dimensionality on the perturbation pressure and vertical velocity in cumulus convection. Part II: Comparison of theoretical and numerical solutions and fully dynamical simulations. J. Atmos. Sci., 73, 1455-1480, https://doi.org/10.1175/JAS-D-15-0041.1.

_- 2017: An analytic description of the structure and evolution of growing deep cumulus updrafts. J. Atmos. Sci., 74, 809-834, https://doi.org/10.1175/JAS-D-16-0234.1.

— cent rate of moist convective thermals. J. Atmos. Sci., 75, 1699-1719, https://doi.org/10.1175/JAS-D-17-0295.1.

_ - G. Thompson, and V. Tatarskii, 2009: Impact of cloud microphysics on the development of trailing stratiform precipitation in a simulated squall line: Comparison of one- and two-moment schemes. Mon. Wea. Rev., 137, 991-1007, https://doi.org/10.1175/ 2008MWR2556.1.

—, J. M. Peters, W. M. Hannah, A. C. Varble, and S. E. Giangrande, 2020: Thermal chains and entrainment in cumulus updrafts: Part I: Theoretical description. J. Atmos. Sci., 77, 3637-3660, https://doi.org/10.1175/JAS-D-19-0243.1.

Morton, B. R., 1957: Buoyant plumes in a moist atmosphere. J. Fluid Mech., 2, 127-144, https://doi.org/10.1017/S0022112057000038.

_, G. Taylor, and J. S. Turner, 1956: Turbulent gravitational convection from maintained and instantaneous sources. Proc. Roy. Soc. London, 234A, 1-23, https://doi.org/10.1098/RSPA.1956.0011.

Naylor, J., and M. S. Gilmore, 2012: Convective initiation in an idealized cloud model using an updraft nudging technique. Mon. Wea. Rev., 140, 3699-3705, https://doi.org/10.1175/MWRD-12-00163.1.

Nowotarski, C. J., J. M. Peters, and J. P. Mulholland, 2020: Evaluating the effective inflow layer of simulated supercell updrafts. Mon. Wea. Rev., 148, 3507-3532, https://doi.org/ 10.1175/MWR-D-20-0013.1.

Peters, J. M., 2016: The impact of effective buoyancy and dynamic pressure forcing on vertical velocities within two-dimensional updrafts. J. Atmos. Sci., 73, 4531-4551, https://doi.org/10.1175/ JAS-D-16-0016.1.

— C. Nowotarski, and H. Morrison, 2019: The role of vertical wind shear in modulating maximum supercell updraft velocities. J. Atmos. Sci., 76, 3169-3189, https://doi.org/10.1175/JAS-D-190096.1.

- , H. Morrison, W. M. Hannah, A. C. Varble, and S. E. Giangrande, 2020a: Thermal chains and entrainment in cumulus updrafts. Part II: Analysis of idealized simulations. J. Atmos. Sci., 77, 3661-3681, https://doi.org/10.1175/JAS-D-19-0244.1.

_ C. Nowotarski, and G. Mullendore, 2020b: Are supercells resistant to entrainment because of their rotation? J. Atmos. Sci., 77, 1475-1495, https://doi.org/10.1175/JAS-D-19-0316.1.

Romps, D. M., 2010: A direct measure of entrainment. J. Atmos. Sci., 67, 1908-1927, https://doi.org/10.1175/2010JAS3371.1. 
2016: The stochastic parcel model: A deterministic parameterization of stochastically entraining convection. J. Adv. Model. Earth Syst., 8, 319-344, https://doi.org/10.1002/2015MS000537.

— , and Z. Kuang, 2010: Nature versus nurture in shallow convection. J. Atmos. Sci., 67, 1655-1666, https://doi.org/10.1175/ 2009JAS3307.1.

—, and A. B. Charn, 2015: Sticky thermals: Evidence for a dominant balance between buoyancy and drag in cloud updrafts. J. Atmos. Sci., 72, 2890-2901, https://doi.org/10.1175/ JAS-D-15-0042.1.

Rotunno, R., and J. B. Klemp, 1982: The influence of the shearinduced pressure gradient on thunderstorm motion. Mon. Wea. Rev., 110, 136-151, https://doi.org/10.1175/15200493(1982)110<0136:TIOTSI > 2.0.CO;2.

— supercell thunderstorms. J. Atmos. Sci., 42, 271-292, https:// doi.org/10.1175/1520-0469(1985)042<0271:OTRAPO > 2.0.CO;2.

Scorer, R. S., 1957: Experiments on convection of isolated masses in buoyant fluid. J. Fluid Mech., 2, 583-594, https://doi.org/ 10.1017/S0022112057000397.

Sherwood, S. C., D. Hernandez-Deckers, and M. Colin, 2013: Slippery thermals and the cumulus entrainment paradox. J. Atmos. Sci., 70, 2426-2442, https://doi.org/10.1175/JAS-D-12-0220.1.

Siebesma, A. P., and Coauthors, 2003: A large eddy simulation intercomparison study of shallow cumulus convection. J. Atmos. Sci., 60, 1201-1219, https://doi.org/10.1175/1520-0469(2003) $60<1201$ :ALESIS $>2.0$.CO;2.

Simpson, J., and V. Wiggert, 1969: Models of precipitating cumulus towers. Mon. Wea. Rev., 97, 471-489, https://doi.org/10.1175/ 1520-0493(1969)097<0471:MOPCT>2.3.CO;2.

Smith, B. T., R. L. Thompson, J. S. Grams, C. Broyles, and H. E. Brooks, 2012: Convective modes for significant severe thunderstorms in the contiguous United States. Part I: Storm classification and climatology. Wea. Forecasting, 27, 11141135, https://doi.org/10.1175/WAF-D-11-00115.1.

Tarshish, N., N. Jeevanjee, and D. Lecoanet, 2018: Buoyant motion of a turbulent thermal. J. Atmos. Sci., 75, 3233-3244, https:// doi.org/10.1175/JAS-D-17-0371.1.

Thompson, R. L., R. Edwards, J. A. Hart, K. L. Elmore, and P. Markowski, 2003: Close proximity soundings within supercell environments obtained from the Rapid Update Cycle. Wea. Forecasting, 18, 1243-1261, https://doi.org/10.1175/15200434(2003)018<1243:CPSWSE $>2.0$.CO;2.

— C. M. Mead, and R. Edwards, 2007: Effective storm-relative helicity and bulk shear in supercell thunderstorm environments. Wea. Forecasting, 22, 102-115, https://doi.org/10.1175/WAF969.1.

— B B. T. Smith, J. S. Grams, A. R. Dean, and C. Broyles, 2012: Convective modes for significant severe thunderstorms in the contiguous United States. Part II: Supercell and QLCS tornado environments. Wea. Forecasting, 27, 1136-1154, https:// doi.org/10.1175/WAF-D-11-00116.1.

Tian, Y., Z. Kuang, M. S. Singh, and J. Nie, 2019: The vertical momentum budget of shallow cumulus convection: Insights from a Lagrangian perspective. J. Adv. Model. Earth Syst., 11, 113-126, https://doi.org/10.1029/2018MS001451.

Tochimoto, E., K. Sueeki, and H. Niino, 2019: Entraining cape for better assessment of tornado outbreak potential in the warm sector of extratropical cyclones. Mon. Wea. Rev., 147, 913-930, https://doi.org/10.1175/MWR-D-18-0137.1.

Trapp, R. J., G. R. Marion, and S. W. Nesbitt, 2017: The regulation of tornado intensity by updraft width. J. Atmos. Sci., 74, 4199-4211, https://doi.org/10.1175/JAS-D-16-0331.1.

Warner, J., 1970: On steady-state one-dimensional models of cumulus convection. J. Atmos. Sci., 27, 1035-1040, https://doi.org/ 10.1175/1520-0469(1970)027<1035:OSSODM>2.0.CO;2.

Warren, R. A., H. Richter, H. A. Ramsay, S. T. Siems, and M. J. Manton, 2017: Impact of variations in upper-level shear on simulated supercells. Mon. Wea. Rev., 145, 2659-2681, https:// doi.org/10.1175/MWR-D-16-0412.1.

Weisman, M. L., and J. B. Klemp, 1982: The dependence of numerically simulated convective storms on vertical wind shear and buoyancy. Mon. Wea. Rev., 110, 504-520, https://doi.org/ 10.1175/1520-0493(1982)110<0504:TDONSC >2.0.CO;2.

$\longrightarrow$, and 1984: The structure and classification of numerically simulated convective storms in directionally varying wind shears. Mon. Wea. Rev., 112, 2479-2498, https://doi.org/ 10.1175/1520-0493(1984)112<2479:TSACON>2.0.CO;2.

- and R. Rotunno, 2000: The use of vertical wind shear versus helicity in interpreting supercell dynamics. J. Atmos. Sci., 57, 1452-1472, https://doi.org/10.1175/1520-0469(2000)057<1452: TUOVWS $>2.0 . \mathrm{CO} ; 2$.

Zhang, G. J., 2009: Effects of entrainment on convective available potential energy and closure assumptions in convection parameterization. J. Geophys. Res., 114, D07109, https://doi.org/ 10.1029/2008JD010976.

—, and N. A. McFarlane, 1991: Convective stabilization in midlatitudes. Mon. Wea. Rev., 119, 1915-1928, https://doi.org/ 10.1175/1520-0493(1991)119<1915:CSIM > 2.0.CO;2.

—, and — 1995: Sensitivity of climate simulations to the parameterization of cumulus convection in the Canadian Climate Center general-circulation model. Atmos.-Ocean, 33, 407-446, https://doi.org/10.1080/07055900.1995.9649539.

Zhao, M., and P. H. Austin, 2003: Episodic mixing and buoyancysorting representations of shallow convection: A diagnostic study. J. Atmos. Sci., 60, 892-912, https://doi.org/10.1175/15200469(2003)060<0892:EMABSR $>2.0 . \mathrm{CO} ; 2$. 